eISSN 2444-7986

DOI: https://doi.org/10.14201/orl.20953

ARTÍCULO DE REVISIÓN

\title{
REHABILITACIÓN VESTIBULAR EN PERSONAS MAYORES CON DISFUNCIÓN VESTIBULAR
}

\section{Vestibular rehabilitation in older people with vestibular dysfunction}

\author{
Virginia FRANCO-GUTIÉRREZ; Paz PÉREZ- VÁZQUEZ² \\ ${ }^{1}$ Servicio de ORL. Hospital Carmen y Severo Ochoa. Cangas del Narcea. Asturias. España \\ ${ }^{2}$ Servicio de Otorrinolaringología. Hospital de Cabueñes. Gijón. Asturias. españa \\ Correspondencia: virginiafrancogutierrez@yahoo.es
}

Fecha de recepción: 22 de junio de 2019

Fecha de aceptación: 12 de julio de 2019

Fecha de publicación: 14 de julio de 2019

Fecha de publicación del fascículo: 1 de marzo de 2020

Conflicto de intereses: Los autores declaran no tener conflictos de intereses

Imágenes: Los autores declaran haber obtenido las imágenes con el permiso de los pacientes

Política de derechos y autoarchivo: se permite el autoarchivo de la versión post-print (SHERPA/RoMEO)

Licencia CC BY-NC-ND. Licencia Creative Commons Atribución-NoComercial-SinDerivar 4.0 Internacional

Universidad de Salamanca. Su comercialización está sujeta al permiso del editor

RESUMEN: Introducción y objetivo: El mareo y el desequilibrio son síntomas comunes en el anciano y tienen un gran impacto en su calidad de vida. Método: Revisión narrativa. Resultados: Se revisan los principales factores que condicionan la inestabilidad en el anciano, el impacto de las caídas y las exploraciones más rentables desde el punto de vista diagnóstico y terapéutico en estos pacientes. Por último, se incide en la necesidad de un abordaje multidisciplinar destacando la importancia de la rehabilitación vestibular en el tratamiento de estos trastornos. Conclusiones: La inestabilidad en el anciano es de origen multifactorial y no obedece solamente a razones fisiológicas. De ahí la necesidad de identificar los factores precipitantes, tratarlos si es posible y realizar un abordaje multidisciplinar que minimice la morbimortalidad asociada a este proceso y mejorando su calidad de vida. La rehabilitación vestibular puede mejorar significativamente el equilibrio de los ancianos con inestabilidad lo cual conduciría a una reducción significativa de las caídas.

PALABRAS CLAVE: inestabilidad; mareo; caídas; ancianos; rehabilitación vestibular. 


\section{REHABILITACIÓN VESTIBULAR EN PERSONAS MAYORES CON DISFUNCIÓN VESTIBULAR FRANCO-GUTIÉRREZ V Y PÉREZ- VÁZQUEZ P}

SUMMARY: Introduction and objective: Dizziness and imbalance are common symptoms in the elderly and have a great impact on their quality of life. Method: Narrative review. Results: Main factors that condition instability in the elderly and the impact of falls are reviewed, as well as the most profitable tests in diagnosis. Finally, the need to perform a multidisciplinary approach was discussed, highlighting the importance of vestibular rehabilitation in the treatment of these disorders. Conclusions: Instability in the elderly is multifactorial and not only due to physiological reasons. Hence the need to identify precipitating factors, treat them if possible and carry out a multidisciplinary approach that minimizes the morbidity and mortality associated with this process and improve their quality of life. Vestibular rehabilitation can significantly ameliorate balance in the elderly with instability which would lead to a significant reduction in falls.

KEYWORDS: instability; dizziness; falls; elderly; vestibular rehabilitation.

\section{INTRODUCCIÓN}

El equilibrio es un proceso complejo dependiente de numerosos estímulos sensoriales periféricos (propioceptivos, visuales y vestibulares) que se integran centralmente y se combinan con otras funciones neurales tales como el control motor y la cognición para mantener el centro de gravedad dentro de unos límites establecidos [1]. En la senectud, sin embargo, la eficacia de estos mecanismos se deteriora como consecuencia del proceso fisiológico de envejecimiento, que afecta a todos los órganos y sistemas del cuerpo humano [2].

El mareo y el desequilibrio son síntomas comunes en el anciano y tienen un gran impacto en su calidad de vida. El mareo interfiere con las actividades diarias del $30 \%$ de pacientes mayores de 70 años. Su etiología es multifactorial, no sólo consecuencia de un hecho fisiológico, de ahí la importancia de identificar los factores precipitantes, tratarlos si es posible y evitar complicaciones como las caídas que se asocian con una significativa morbimortalidad en el anciano [3].

\section{CAMBIOS FUNCIONALES EN LOS PACIENTES ANCIANOS}

Las alteraciones del equilibrio que pueden aparecer en el anciano como consecuencia del envejecimiento y deterioro fisiológico parejo a la edad suelen denominarse presbivértigo. Aunque son multifactoriales, reconocen una participación importante del menoscabo del sistema vestibular. El deterioro gradual del sistema vestibular es simétrico y comienza en la edad media de la vida, pero la compensación vestibular evita que se manifieste hasta edades más avanzadas. Desde el punto de vista histológico, el envejecimiento vestibular se manifiesta por pérdida y degeneración de células sensoriales, de neuronas ganglionares y nucleares vestibulares y de fibras aferentes [4] (Tabla 1).

Tabla 1. Principales cambios histológicos asociados al envejecimiento en el sistema vestibular

- Disminución de células ciliadas, más precoz en crestas que en máculas y en las de tipo I

- Cambios histológicos degenerativos en las células ciliadas

- Cambios degenerativos en las otoconias (más marcado en el utrículo)

- Degeneración de las aferencias vestibulares primarias (más marcado en las aferencias irregulares que en las regulares

- Descenso del número y densidad de las neuronas del ganglio de Scarpa y en el nervio vestibular (más en la rama superior que en la inferior)

- Descenso del número de neuronas en el complejo nuclear vestibular

- Aumento de las neuronas gigantes en los núcleos vestibulares

- Disminución del volumen del cerebelo, de la densidad de las células de Purkinje y de la sustancia blanca del lóbulo flóculo-nodular 


\section{REHABILITACIÓN VESTIBULAR EN PERSONAS MAYORES CON DISFUNCIÓN VESTIBULAR FRANCO-GUTIÉRREZ V Y PÉREZ- VÁZQUEZ P}

Desde el punto de vista funcional el reflejo vestíbulo-ocular (RVO) comienza a declinar por encima de los 75 años y se ha descrito la disminución de la ganancia y la constante de tiempo del RVO, especialmente durante la estimulación a altas frecuencias. También disminuye la capacidad de adaptar la ganancia del RVO, lo que afectaría a la capacidad del sistema para ajustarse a una pérdida de función vestibular o a otros estímulos estresantes $[5,6]$.

Tan importantes como el deterioro del sistema vestibular para el equilibrio en los ancianos lo son toda una serie de condicionantes multiorgánicos que deben tenerse en cuenta (Figura 1):

- Déficits visuales, que pueden ser ignorados porque se instauran de forma progresiva [7]. La agudeza visual, la capacidad de acomodación, así como la adaptación a la oscuridad disminuyen con la edad [8,9]. Por otro lado, existen otros trastornos frecuentes en los ancianos como cataratas, glaucoma, degeneración macular o alteraciones perceptivas de la profundidad [10].

- Disminución de la sensibilidad propioceptiva $[11,12]$.

- Alteraciones en el sistema cardiovascular (hipotensión ortostática, HTA, arterioesclerosis, arritmias cardiacas).

- Enfermedades metabólicas (como la diabetes mellitus, que se asocia a neuropatías y retinopatía y favorece la arteriosclerosis).

- Alteraciones en el sistema musculoesquelético: descalcificación ósea, rigidez articular, sarcopenia y enlentecimiento de las respuestas motoras $[4,13]$.

- Deterioro cognitivo o alteración del estado de ánimo [14].

- Alteraciones en el sistema nervioso central (SNC): Las lesiones de sustancia blanca frecuentes en pacientes mayores de 60 años pueden producir un daño en romper las conexiones cortico-subcorticales implicadas

\section{Alteraciones en el SNC \\ Lesiones blancas \\ Activación cortical}

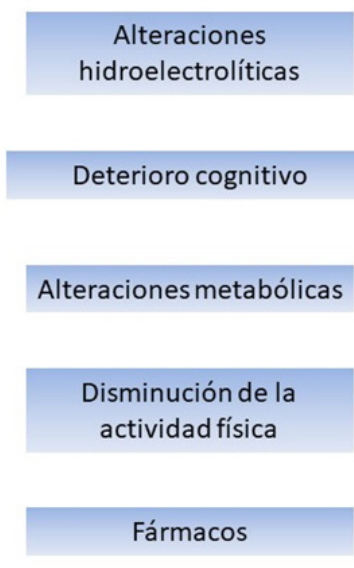

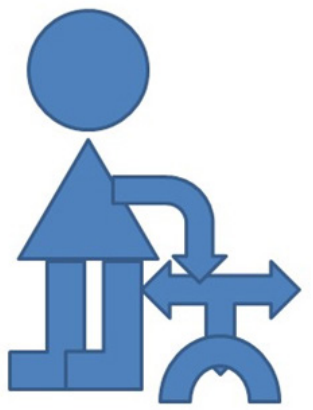

Alteraciones en el sistema vestibular

\section{Alteraciones en el sistema musculoesquelético \\ Sarcopenia, descalcificación ósea, rigidez articular, enlentecimiento de las respuestas motoras}

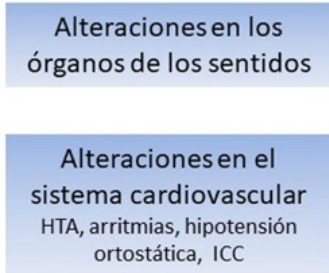

Figura 1: Condicionantes del equilibrio en el anciano 


\section{REHABILITACIÓN VESTIBULAR EN PERSONAS MAYORES CON DISFUNCIÓN VESTIBULAR FRANCO-GUTIÉRREZ V Y PÉREZ- VÁZQUEZ P}

en el control motor y del equilibrio [15]. También se produce una mayor activación de las estructuras corticales en el anciano, pues al tener reducido el input somatosensorial precisan apoyarse en otras estructuras corticales para la integración sensorial [16].

- Disminución de la actividad física

- Toma de fármacos, con posibles efectos sedantes, hipotensores ortostáticos [13].

Todos estos factores determinan modificaciones tanto en la estática como en la marcha de los ancianos.

\section{CONTROL POSTURAL Y DINÁMICA DE LA MARCHA EN EL ANCIANO}

El declive del control postural ya es evidente a edades no muy tardías si sometemos a un individuo a una situación que suponga un desafío para su equilibrio y se acentúa por cada década de la vida.

Los ancianos doblan la velocidad de oscilación cuando, situados sobre una superficie firme, proceden a cerrar los ojos. Esto se ha relacionado con la degeneración del sistema vestibular [17].

También pueden emplear secuencias de activación muscular y estrategias inadecuadas para mantener la estabilidad [18].

La disminución de los input visual o propioceptivo del pie/tobillo en el anciano conlleva una alteración del equilibrio que se incrementa con la disminución de la fuerza muscular [19]. Existe controversia acerca de si la repercusión es mayor cuando disminuye el input propioceptivo o cuando lo hace el visual [20,21], lo que sí está claro es que el anciano confía mucho en la visión para mantener su estabilidad [22]. De igual modo, presentan menor capacidad para adaptarse a nuevas informaciones sensoriales [21].

En la marcha también influye la función de los sistemas sensoriales y las reservas cognitiva y locomotora [23]. El control sensorial de la marcha es especialmente importante en el contexto de la marcha lenta y menos relevante en la marcha rápida. Respecto a la dinámica de la marcha, la velocidad disminuye a partir de los 60 años un 1\% por cada año [24]. Normalmente la marcha es automática y requiere poca atención. Sin embargo, con el envejecimiento, el control central de la marcha aumenta, pero como simultáneamente la interacción sensorial central está dañada, el paciente refiere sensación de mareo al caminar [25].

Lo anteriormente expuesto tiene una serie de implicaciones prácticas:

- Los criterios de normalidad deben ser ajustados cuando se examina un anciano.

- Los hallazgos clínicos relacionados con la edad dan testimonio de los mecanismos subyacentes responsables del deterioro fisiológico del equilibrio en el anciano.

- Cualquier enfermedad adicional que afecte al equilibrio tendrá mayor impacto en el anciano. Igualmente, el proceso de compensación típicamente basado en la sustitución y la compensación central se verá comprometido [26].

\section{RIESGO DE CAIIDAS}

Las alteraciones del equilibrio y la marcha se han asociado con un incremento en el riesgo de caídas [27]. En adultos de 65 o más años la prevalencia anual de caídas es del $28 \%$ y la tasa de caídas y la prevalencia de los factores de riesgo para las mismas se incrementan de forma acusada a partir de los 70 años [12,28].

Las caídas se asocian con una importante morbimortalidad en el anciano: constituyen la causa más frecuente de muerte accidental y daño accidental no fatal en las personas de 65 años y mayores, siendo responsables del $55,8 \%$ de muertes accidentales [12]. Casi un tercio de las personas que se caen tres o más veces al año precisan hospitalización, ingreso en un centro residencial o mueren al año siguiente [29]. La mitad de los ancianos que se caen tienen caídas recurrentes y el 50\% vuelve caer en el mismo año, por lo que la caída es un 


\section{REHABILITACIÓN VESTIBULAR EN PERSONAS MAYORES CON DISFUNCIÓN VESTIBULAR FRANCO-GUTIÉRREZ V Y PÉREZ- VÁZQUEZ P}

factor de riesgo para sufrir una nueva caída. Más de un $70 \%$ tienen consecuencias clínicas como fracturas, esguinces, heridas...y más de la mitad presentan secuelas posteriormente [30]. El 50\% de las personas que sufren una fractura por una caída no recuperan el nivel funcional previo y una de cada 10 caídas genera lesiones graves.

Las consecuencias de las caídas repercuten sobre la calidad de vida de quienes las padecen, destacando el síndrome del temor a caerse, que puede suponer el inicio de la discapacidad en el anciano [13].

Tienen pues, una importante repercusión económica. En EEUU se ha estimado que los costes médicos asociados a caídas son superiores a 19 billones de dólares anuales [31].

Las mujeres son más proclives a relatar caídas y complicaciones relacionadas con las mismas que los hombres [7].

\section{EVALUACIÓN EN PACIENTES ANCIANOS CON INESTABILIDAD}

\section{A) Exploración vestibular}

Las pruebas que evalúan el RVO muestran pocos cambios con la edad y pueden superponerse y enmascarar o limitar el diagnóstico de patología vestibular pues, como ya hemos señalado, el RVO comienza a declinar por encima de los 75 años $[3,5,6]$ :

- Las pruebas calóricas y rotatorias pueden mostrar datos de disfunción vestibular bilateral atribuible a la edad, generalmente de escasa cuantía $[6,13,32]$.

- V-HIT: las ganancias a nivel de todos los conductos semicirculares se mantienen dentro de límites normales hasta edades avanzadas. En los pacientes de más de 80 años se produce una disminución de la ganancia, pero sin que se haya podido relacionar con manifestaciones clínicas. Recientemente se ha descrito una aplicación de este dispositivo para la valoración del equilibrio en el anciano: la presencia de sacadas de refijación de gran amplitud y escasa latencia se ha asociado con la incapacidad para la realización de la prueba de tándem; reflejarían la estrategia de estabilización de la mirada que compensaría un RVO y una función vestibular deficientes [33].

En relación con la exploración del reflejo vestíbulo cólico, dado que los potenciales evocados miogénicos vestibulares (VEMP) pueden estar ausentes en pacientes mayores de 60 años no tendrían mucho rendimiento diagnóstico. Se ha objetivado un aumento en el umbral y disminución de la amplitud de respuesta en relación con el envejecimiento [34].

\section{B) VALORACión DEL RIESGo DE CAÍDAS}

La tasa de caídas y la prevalencia de los factores de riesgo para las mismas se incrementan de forma acusada a partir de los 70 años [28].

La valoración de un anciano con caídas es compleja y va más allá de una mera exploración vestibular.

Se debe recoger específicamente la historia de caídas. Es sumamente importante diferenciar las caídas de los traspiés y recoger si conllevaron o no daño que precisara asistencia médica, de qué tipo y el número de caídas en el último año.

También se debe investigar la capacidad de realizar las actividades básicas e instrumentales de la vida diaria.

Es importante inspeccionar las articulaciones en busca de deformidades, tumefacción, inestabilidad y limitación en el rango de movimiento activo y pasivo, especialmente en las extremidades inferiores y la columna vertebral.

Debe valorarse la postura, particularmente la cifosis excesiva, que es habitual en ancianos y puede modificar el centro de gravedad corporal. El desplazamiento anterior cefálico junto con la extensión cervical limitada también altera el centro 


\section{REHABILITACIÓN VESTIBULAR EN PERSONAS MAYORES CON DISFUNCIÓN VESTIBULAR FRANCO-GUTIÉRREZ V Y PÉREZ- VÁZQUEZ P}

de gravedad y limita el campo visual periférico funcional, contribuyendo al desequilibrio.

Debe realizarse una exploración neurológica en busca alteraciones sensoriales, fundamentalmente propioceptivas y de pérdida de fuerza. También es necesario evaluar coordinación, tono muscular, temblores, cognición y depresión (mediante cuestionarios validados) [7].

La valoración debe incluir un examen de la marcha, que debería realizarse sin que el paciente sea consciente de dicha evaluación. Las pruebas más útiles para ello serían:

- La prueba de timed up and go (valora el tiempo que tarda un paciente en levantarse de una silla, caminar 3 metros, dar la vuelta y volverse a sentar). Si tarda veinte o más segundos en realizarla se considera que el anciano tiene un elevado riesgo de caída.

- La velocidad de la marcha en función al tiempo cronometrado que tarda el paciente en recorrer 3- $5 \mathrm{~m}$ [10]. Valores de $0,8 \mathrm{~m} / \mathrm{s}$ o inferiores se consideran patológicos.

- La capacidad de levantarse de una silla, que es un screening funcional excelente pues valora simultáneamente fortaleza proximal, equilibrio y coordinación [7]. Se valora de forma cualitativa.

Durante la exploración del reflejo vestíbulo espinal es habitual, a partir de los 70-80 años, objetivar un aumento de las oscilaciones con tendencia a la caída en la prueba de Romberg, así como un incremento progresivo de la amplitud de las oscilaciones laterales en la prueba de Unterberger [13]. Se debe valorar el Romberg en tándem y si el paciente es capaz de mantener su estabilidad cuando permanece de pie apoyado sobre una sola extremidad inferior.

El resultado de todas estas pruebas tiene implicaciones desde el punto de vista de la rehabilitación pues nos ayudan a determinar qué movimientos son difíciles de realizar para el paciente y así orientar mejor el tratamiento a realizar.
Una prueba de importancia en la valoración del riesgo de caídas es el Short Physical Performance Battery (SPPB). Es sencillo y aporta gran información acerca del estado general de salud, equilibrio y fuerza [35]. Consiste en la realización de tres pruebas:

- Equilibrio (en tres posiciones: pies juntos, semitándem y tándem)

- Velocidad de la marcha (sobre 2.4 o 4 metros)

- Levantarse y sentarse en una silla cinco veces.

La puntuación y valoración del resultado total del SPPB resulta de la suma de los tres apartados y oscila entre 0 (peor) y 12 (excelente). Una puntuación por debajo de 10 indica fragilidad, discapacidad y un elevado riesgo de caídas. Los valores normativos para la población española se han establecido en diversos estudios de cohortes poblacionales y en atención primaria [36].

\section{C) Estudios POSTURográficos:}

Se ha descrito un menor control del equilibrio asociado a la edad en la posturografía y que la alteración en dos de los tres inputs sensoriales (vestibular, visual y somatosensorial) tiene una mayor repercusión en los ancianos. Obtienen peores resultados en las condiciones 4, 5 y 6 del test de organización sensorial (SOT) pero no en las condiciones 1,2 y 3 . Por lo tanto, parece que hacen peor uso de la información visual y vestibular, pero su empleo de la información somatosensorial no disminuye a lo largo del tiempo. Tampoco la tolerancia al conflicto visual es menor, aunque las caídas sean más frecuentes durante el SOT.

Se ha constatado que los pacientes ancianos muestran un control direccional anteroposterior sustancialmente más pobre que el lateral. Dado que la mayoría de los movimientos de la vida diaria se realizan en el plano anteroposterior (caminar, estar de pie, acostarse, etc.), una menor eficiencia en el movimiento del centro de gravedad en el plano anteroposterior se asocia con un mayor riesgo de caídas [37]. 


\section{REHABILITACIÓN VESTIBULAR EN PERSONAS MAYORES CON DISFUNCIÓN VESTIBULAR FRANCO-GUTIÉRREZ V Y PÉREZ- VÁZQUEZ P}

Se ha descrito que los parámetros más importantes para identificar a pacientes ancianos inestables con riesgo alto de caídas repetidas son las puntuaciones bajas en el equilibrio global medio del SOT, el mal control direccional con desplazamiento del centro de gravedad corporal y los límites de estabilidad reducidos, así como los valores altos obtenidos en el cuestionario short FES-I score [38].

\section{MANEJO TERAPÉUTICO}

En el caso de que el paciente presente un trastorno vestibular concreto, deberá realizarse el tratamiento oportuno del mismo, adaptándolo a las condiciones y limitaciones propias de la edad que se han comentado previamente.

En este capítulo nos centraremos en el tratamiento específico de los condicionantes de los pacientes ancianos, especialmente centrado en presbivértigo con inestabilidad y con riesgo de caídas. El objetivo fundamental del tratamiento será mejorar su estabilidad para reducir la dependencia de terceras personas en la realización de sus actividades cotidianas, disminuir el riesgo de caídas y, en consecuencia, las complicaciones derivadas de estas.

El abordaje de estos pacientes debe ser personalizado y multidisciplinar y debe incluir:

1. Tratamiento de las patologías de base, si las hubiere

2. Información al paciente y familia acerca del riesgo de caídas y de las estrategias para la prevención de estas, actuando sobre los factores de riesgo intrínsecos y extrínsecos.

\section{A) Actuación Sobre factores de RIESgo EXTRÍNSECOS}

Existe una importante evidencia que apoya la valoración y modificación del ambiente domiciliario del paciente como parte de un programa multifactorial de prevención de riesgo de caídas [7] (tabla 2).

Ediciones Universidad de Salamanca / @D@
Tabla 2. Consejos útiles para modificar el ambiente domiciliario del anciano

- Evitar lugares poco iluminados o la luz directa y deslumbrante

- Interruptores accesibles

- Iluminación adecuada en todas las estancias (especialmente en el dormitorio y el cuarto de baño)

- Las luces asociadas a sensores de movimiento pueden ser útiles

- Evitar suelos excesivamente resbaladizos (mojados, encerados...), alfombras y felpudos deteriorados u objetos que obstaculicen el paso

- Escaleras dotadas de pasamanos con escalones en buenas condiciones y a una altura adecuada

- Evitar asientos inestables, bajos, sin respaldo o reposabrazos.

- Estantes accesibles

- Interruptores del gas correctamente señalizados.

- Cama de altura adecuada (cuando el paciente esté sentado en ella la rodilla quede a $90^{\circ}$ )

- Evitar los colchones blandos

- Inodoro de asiento alto, con al menos un asidero de 75 $\mathrm{cm}$ de altura a uno de los lados

- Disponer de asideros en la pared del cuarto de baño

- Disponer de asiento/ banco de ducha para el baño

- Sustituir toalleros por barras de apoyo antideslizantes bien anclados

- Bandas antideslizantes en el suelo del baño

- Retirar cerrojos de las puertas y facilitar que se pueda abrir desde ambos lados.

- La puerta debe abrir hacia fuera.

- Emplear calzado cerrado, confortable, bien adaptado al pie y ligero.

- Empleo de utensilios de ayuda para la movilidad (bastones, andadores...) adecuadamente adaptados.

B) Actuación sobre Factores de Riesgo INTRÍNSECOS

- Ejercicio físico moderado dentro de sus posibilidades y vida lo más activa posible, recomendando el empleo de apoyos en caso de inseguridad/ inestabilidad manifiesta. Se deben incorporar actividades que mantengan o incrementen la flexibilidad, resistencia, fortaleza y equilibrio. El ejercicio físico tiene efectos consistentes en la reducción de caídas en pacientes ancianos sanos cuando se 


\section{REHABILITACIÓN VESTIBULAR EN PERSONAS MAYORES CON DISFUNCIÓN VESTIBULAR FRANCO-GUTIÉRREZ V Y PÉREZ- VÁZQUEZ P}

prescriben y completan a la adecuada progresión e intensidad [39].

- Ajustar la medicación dentro de lo posible. No dar sedantes vestibulares.

- Tratamiento específico de las enfermedades coexistentes.

- Protocolos de rehabilitación vestibular.

La rehabilitación vestibular estará encaminada a mejorar el control postural para disminuir la inestabilidad y las caídas, mejorando la calidad de vida del paciente. Puede ser instrumental (realizada bajo control en el centro hospitalario), estar basada en ejercicios domiciliarios, o combinar ambos protocolos. Se ha observado que los mejores resultados se obtienen combinando las sesiones de rehabilitación vestibular instrumental con un programa de ejercicios en casa, ya que estos últimos refuerzan el tratamiento que realizamos en la clínica y de ello depende en gran medida el proceso de consolidación y mejora de los resultados obtenidos [40].

\section{EJERCICIOS DOMICILIARIOS}

El protocolo de ejercicios domiciliarios debe ser atractivo e individualizado, adaptándolo a la situación del paciente. Se debe iniciar progresivamente, con ejercicios que garanticen la entrada de información a partir de todas las vías posibles, adaptando la dificultad, el tiempo y el tipo de ejercicios a la evolución del paciente. Se aumentará escalonadamente la participación en el entrenamiento de escenarios reales de complejidad creciente (mediante videoconsolas, acudiendo a ambientes en los que se produce conflicto sensorial, practicando deportes o bailes). Es importante controlar la evolución y la adherencia al tratamiento, pues de ella depende el éxito del programa.

En los ancianos con inestabilidad frecuentemente nos encontramos con un síndrome vestibular crónico que tiene síntomas desencadenados por los movimientos o el conflicto visual, incluso trastornos psicológicos derivados de las situaciones anteriores. En estos casos probablemente sean más útiles los ejercicios progresivos de habituación (para la intolerancia al movimiento) o de entrenamiento con entornos móviles o estímulos optocinéticos. También es útil en estos casos potenciar el control postural con ejercicios de Tai Chi. El entrenamiento de la preprogramación central de los movimientos oculares y los ejercicios de sustitución para la estabilización de la postura pueden ser muy útiles en los casos particulares de síndrome vestibular crónico en el que se constate un déficit vestibular bilateral.

La importancia de los ejercicios domiciliarios como tratamiento único se entiende si tenemos en cuenta que la estructura organizativa y la dinámica asistencial de la sanidad pública impiden asumir el tratamiento hospitalario de todos los posibles pacientes ancianos subsidiarios de rehabilitación vestibular. Además, las características de los problemas que tratamos, que generalmente requieren modificaciones en la vida del paciente, hacen interesante introducir estas pautas en su vida diaria [41]. Su éxito depende de que se realicen los ejercicios varias veces al día y de forma continuada a lo largo del tiempo.

\section{REHABILITACIÓN INSTRUMENTAL}

Existe evidencia de que los pacientes ancianos con alteración en los input vestibular y visual o con límites de estabilidad reducidos se beneficiarán más de la rehabilitación mediante posturografía, mientras que los pacientes con mala tolerancia al conflicto visual se beneficiarían más de rehabilitación mediante estímulo optocinético [42]. Los pacientes con una prueba de timed up and go patológica obtendrían mejores resultados del entrenamiento de la marcha [43].

También se han obtenido buenos resultados en la rehabilitación de pacientes ancianos inestables empleando dispositivos vibrotáctiles, que se comportan como posturógrafos portátiles, permitiendo evaluar el equilibrio de los pacientes y detectar el perfil de situaciones en las cuales el mantenimiento de la estabilidad está más comprometido, de forma que podamos proceder a su 


\section{REHABILITACIÓN VESTIBULAR EN PERSONAS MAYORES CON DISFUNCIÓN VESTIBULAR FRANCO-GUTIÉRREZ V Y PÉREZ- VÁZQUEZ P}

rehabilitación. Estos dispositivos están programados de tal modo que producen una vibración cuando el paciente se desplaza excesivamente en cualquiera de las cuatro direcciones (anterior, posterior, derecha e izquierda) y este feed-back vibratorio ayuda al enfermo a corregir y disminuir el desplazamiento de su centro de gravedad. Dado que permite el entrenamiento del equilibrio en situaciones dinámicas, es especialmente útil en pacientes que presentan limitaciones en la marcha y durante el desplazamiento [44].

Sobre la duración de la rehabilitación instrumental, no existe una evidencia científica de cuáles son la frecuencia, la intensidad y el tiempo necesarios para una óptima recuperación. En un estudio en pacientes ancianos con inestabilidad debido a una hipofunción vestibular unilateral crónica y sometidos a rehabilitación mediante posturografía se sugiere que, dado que se obtienen ya importantes mejoras con sólo cinco sesiones, ese sería el número óptimo de las mismas para este tipo de pacientes. Sin embargo, puntualizan que los pacientes con límites de estabilidad reducidos y por tanto gran riesgo de caídas, son candidatos a un mayor número de sesiones [45]. Estos pacientes, especialmente si son varones, son más proclives a abandonar los programas de rehabilitación [46].

Existen datos que avalan el beneficio para la mejora del equilibrio del yoga, del Tai chi o de practicar con dispositivos lúdicos como la wii fit [10].

Por último, existen algunos estudios que avalan la utilidad de la psicoterapia. Mediante el abordaje cognitivo conductual los pacientes aprenden acerca del mareo, el sistema vestibular, discuten su experiencia con el mareo y sus reacciones al mismo y aprenden a exponerse a situaciones que desencadenan sus síntomas, combinándolos con ejercicios y técnicas de relajación [47].

Lo que está claro es que sea cual sea la modalidad de rehabilitación vestibular elegida, ésta puede mejorar decisivamente el equilibrio de los ancianos con inestabilidad, lo que conduciría a una reducción significativa de las caídas [48].

\section{CONCLUSIONES}

La inestabilidad en el anciano es de origen multifactorial y no obedece solamente a razones fisiológicas. De ahí la necesidad de identificar los factores precipitantes, tratarlos si es posible y realizar un abordaje multidisciplinar que minimice la morbimortalidad asociada a este proceso y mejore su calidad de vida. La rehabilitación vestibular puede mejorar significativamente el equilibrio de los ancianos con inestabilidad lo cual conduciría a una reducción significativa de las caídas.

\section{AGRADECIMIENTOS}

A los Dres Santos Pérez, Faraldo García, Soto Varela, y Rossi Izquierdo por su inestimable ayuda y guía. Su gran trabajo ha sido la base de esta revisión y de nuestro proceder en la práctica diaria con estos pacientes.

\section{BIBLIOGRAFÍA}

1. Pollock AS, Durward BR, Rowe PJ, Paul JP. What is balance? Clin Rehabil. 2000;14:402-6.

2. Bracchi E, Rizzo S, Longari F, Bernardini M, Bizzotti C, Frenguelli A. Balance disorders in the elderly. Acta Otorhinolaryngol Ital. 2002;22:263-7.

3. Jahn K. The Aging Vestibular System: Dizziness and Imbalance in the Elderly. Adv Otorhinolaryngol. 2019;82:143-9.

4. Eibling D. Balance Disorders in Older Adults. Clin Geriatr Med. 2018;34:175-81.

5. Ishiyama G. Imbalance and vertigo: the aging human vestibular periphery. Semin Neurol. 2009;29:491-9.

6. Baloh RW, Jacobson KM, Socotch TM. The effect of aging on visual-vestibuloocular responses. Exp Brain Res. 1993;95:509-16.

7. Cuevas-Trisan R. Balance Problems and Fall Risks in the Elderly. Clin Geriatr Med. 2019;35:173-83.

8. Spooner JW, Sakala SM, Baloh RW. Effect of aging on eye tracking. Arch Neurol. 1980;37:575-6. 


\section{REHABILITACIÓN VESTIBULAR EN PERSONAS MAYORES CON DISFUNCIÓN VESTIBULAR FRANCO-GUTIÉRREZ V Y PÉREZ- VÁZQUEZ P}

9. Sharpe JA, Sylvester TO. Effect of aging on horizontal smooth pursuit. Invest Ophthalmol Vis Sci. 1978;17:465-8.

10. Whitney SL, Marchetti GF. Physical therapy management of the older person with vestibular rehabilitation. In Herdman SJ, Clendaniel RA, editors. Vestibular rehabilitation. 4aEdition. Philadelphia: F.A. Davis Company; 2014;p.480503.

11. Robbins S, Waked E, McClaran J. Proprioception and stability: foot position awareness as a function of age and footwear. Age Ageing. 1995;24:67-72.

12. Patel M, Magnusson M, Kristinsdottir E, Fransson PA. The contribution of mechanoreceptive sensation on stability and adaptation in the young and elderly. Eur J Appl Physiol. 2009;105:167-73.

13. Faraldo García A. Paciente con presbivértigo y riesgo de caídas. En Rosssi- Izquierdo M, Soto Varela A, Santos Pérez S, editores. Rehabilitación vestibular [ Internet]. Santiago de Compostela: Sociedad Gallega de Otorrinolaringología; 2016 [acceso 8 de junio de 2018].p. 195- 202. Disponible en: http://www.sgorl.org/images/Ponencias/ Ponencia_SGORL_Rehabilitación_Vestibular_. pdf.

14. Kearney FC, Harwood RH, Gladman JR, Lincoln $\mathrm{N}$, Masud T. The relationship between executive function and falls and gait abnormalities in older adults: a systematic review. Dement Geriatr Cogn Disord. 2013;36:20-35.

15. Shen DC, Wu SL, Shi YZ, Wang S, Zhang YM, Wang CX. The correlation between white matter hyperintensity and balance disorder and fall risk: An observational, prospective cohort study. Chronic Dis Transl Med. 2016; 15:173-80.

16. Lin CC, Barker JW, Sparto PJ, Furman JM, Huppert TJ. Functional near-infrared spectroscopy (fNIRS) brain imaging of multi-sensory integration during computerized dynamic posturography in middle-aged and older adults. Exp Brain Res. 2017;235:1247-56.

17. Pyykkö I, Jäntti $\mathrm{P}$, Aalto $\mathrm{H}$. Postural control in elderly subjects. Age Ageing. 1990;19:215-21.
18. Manchester D, Woollacott M, Zederbauer-Hylton $\mathrm{N}$, Marin O.Visual, vestibular and somatosensory contributions to balance control in the older adult. J Gerontol. 1989;44:118-27.

19. Judge JO, King MB, Whipple R, Clive J, Wolfson LI. Dynamic balance in older persons: effects of reduced visual and proprioceptive input. J Gerontol A Biol Sci Med Sci. 1995;50:263-70.

20. Shumway-Cook A, Woollacott M, Kerns KA, Baldwin M. The effects of two types of cognitive tasks on postural stability in older adults with and without a history of falls. J Gerontol A Biol Sci Med Sci. 1997;52:232-40.

21. Panzer VP, Bandinelli S, Hallett M. Biomechanical assessment of quiet standing and changes associated with aging. Arch Phys Med Rehabil. 1995;76:151-7.

22. Jeka JJ, Allison LK, Kiemel T. The dynamics of visual reweighting in healthy and fall-prone older adults. J Mot Behav. 2010;42:197-208.

23. Verghese J, Ayers E, Barzilai N, Bennett DA, Buchman AS, Holtzer R, et al. Motoric cognitive risk syndrome: Multicenter incidence study. Neurology. 2014;83:2278-84.

24. Osoba MY, Rao AK, Agrawal SK, Lalwani AK. Balance and gait in the elderly: A contemporary review. Laryngoscope Investig Otolaryngol. 2019;4:143-53.

25. Zwergal A, Linn J, Xiong G, Brandt T, Strupp M, Jahn K. Aging of human supraspinal locomotor and postural control in fMRI. Neurobiol Aging. 2012;33:1073-84.

26. Bronstein A, Lempert T. Dizziness, imbalance and falls in the elderly. En: Bronstein A, Lempert T, editors. Dizziness. A practical approach to diagnosis and management 2a Edition. Cambridge (United Kingdom): Cambridge University Press. 2017.p. 147-66.

27. Tinetti ME, Speechley M, Ginter SF. Risk factors for falls among elderly persons living in the community. N Engl J Med. 1988;319:1701-7.

28. Shaw FE, Bond J, Richardson DA, Dawson P, Steen IN, McKeith IG, et al. Multifactorial intervention after a fall in older people with cognitive 


\section{REHABILITACIÓN VESTIBULAR EN PERSONAS MAYORES CON DISFUNCIÓN VESTIBULAR FRANCO-GUTIÉRREZ V Y PÉREZ- VÁZQUEZ P}

impairment and dementia presenting to the accident and emergency department: randomised controlled trial. BMJ. 2003;326:73.

29. Lord SR, McLean D, Stathers G. Physiological factors associated with injurious falls in older people living in the community. Gerontology. 1992;38:338-46.

30. Panel on Prevention of Falls in Older Persons, American Geriatrics Society and British Geriatrics Society. Summary of the Updated American Geriatrics Society/British Geriatrics Society clinical practice guideline for prevention of falls in older persons. J Am Geriatr Soc. 2011;59:148-57.

31. Stevens JA, Corso PS, Finkelstein EA, Miller TR. The costs of fatal and non-fatal falls among older adults. Inj Prev. 2006;12(5):290-5.

32. Peterka RJ, Black FO, Schoenhoff MB. Age-related changes in human vestibulo-ocular reflexes: sinusoidal rotation and caloric tests. J Vestib Res. 1990-1991;1(1):49-59.

33. Xie Y, Anson ER, Simonsick EM, Studenski SA, Agrawal Y. Compensatory Saccades Are Associated With Physical Performance in Older Adults: Data From the Baltimore Longitudinal Study of Aging. Otol Neurotol. 2017;38:373-8.

34. Krager R. Assessment of vestibular function in elderly patients. Curr Opin Otolaryngol Head Neck Surg. 2018;26:302-6.

35. McMurdo ME, Gaskell A. Dark adaptation and falls in the elderly.Gerontology. 1991;37(4):221-4.

36. Cabrero-García J, Muñoz-Mendoza CL, Cabañero-Martínez MJ, González-Llopís L, RamosPichardo JD, Reig-Ferrer A. Valores de referencia de la Short Physical Performance Battery para pacientes de 70 y más años en atención primaria de salud. Aten Primaria. 2012;44:540-8.

37. Soto-Varela A, Rossi-Izquierdo M, Faraldo-García A, Vaamonde-Sánchez-Andrade I, Gayoso-Diz P, Del-Río-Valeiras Met al. Balance Disorders in the Elderly: Does Instability Increase Over Time? Ann Otol Rhinol Laryngol. 2016;125(7):550-8.

38. Soto-Varela A, Faraldo-García A, Rossi-Izquierdo M, Lirola-Delgado A, Vaamonde-SánchezAndrade I, del-Río-Valeiras $\mathrm{M}$, et al. Can we predict the risk of falls in elderly patients with instability? Auris Nasus Larynx. 2015;42:8-14.

39. Sherrington C, Michaleff ZA, Fairhall N, Paul SS, Tiedemann A, Whitney J, et al. Exercise to prevent falls in older adults: an updated systematic review and meta-analysis. Br J Sports Med. 2017;51(24):1750-8.

40. Hall CD, Herdman SJ, Whitney SL, Cass SP, Clendaniel RA, Fife TD, et al. Vestibular Rehabilitation for Peripheral Vestibular Hypofunction: An Evidence-Based Clinical Practice Guideline: From the American Physical Therapy Association Neurology Section. J Neurol Phys Ther. 2016 Apr;40(2):124-55.

41. Gabriel Trinidad Ruíz. Ejercicios de rehabilitiación domiciliaria. En Rosssi- Izquierdo M, Soto Varela A, Santos Pérez S, editores. Rehabilitación vestibular [ Internet]. Santiago de Compostela: Sociedad Gallega de Otorrinolaringología;2016 [acceso 8 de junio de 2018].p. 169- 77. Disponible en: http://www.sgorl.org/images/Ponencias/ Ponencia_SGORL_Rehabilitación_Vestibular_. pdf.

42. Rossi-Izquierdo M, Santos-Pérez S, Soto-Varela A. What is the most effective vestibular rehabilitation technique in patients with unilateral peripheral vestibular disorders? Eur Arch Otorhinolaryngol. 2011; 268:1569-74.

43. Rossi-Izquierdo M, Gayoso-Diz P, SantosPérez S, Del-Río-Valeiras M, Faraldo-García A, Vaamonde-Sánchez-Andrade I, et al. Prognostic factors that modify outcomes of vestibular rehabilitation in elderly patients with falls. Aging Clin Exp Res. 2019 Apr 11. doi: 10.1007/s40520-01901185-8. [Epub ahead of print].

44. Basta D, Rossi-Izquierdo M, Soto-Varela A, Greters ME, Bittar RS, Steinhagen-Thiessen E,et al. Efficacy of a vibrotactile neurofeedback training in stance and gait conditions for the treatment of balance deficits: a double-blind, placebo-controlled multicenter study. Otol Neurotol. 2011; 32:1492-9.

45. Rossi-Izquierdo M, Santos-Pérez S, Rubio-Rodríguez JP, Lirola-Delgado A, Zubizarreta-Gutiérrez 


\section{REHABILITACIÓN VESTIBULAR EN PERSONAS MAYORES CON DISFUNCIÓN VESTIBULAR FRANCO-GUTIÉRREZ V Y PÉREZ- VÁZQUEZ P}

A, San Román-Rodríguez E, et al. What is the optimal number of treatment sessions of vestibular rehabilitation? Eur Arch Otorhinolaryngol. 2014; 271:275-80.

46. Soto-Varela A, Faraldo-García A, Del-Río-Valeiras M, Rossi-Izquierdo M, Vaamonde-SánchezAndrade I, Gayoso-Diz P, et al. Adherence of older people with instability in vestibular rehabilitation programmes: prediction criteria. J Laryngol Otol. 2017; 131:232-8.
47. Brosel S, Strupp M. The Vestibular System and Ageing. Subcell Biochem. 2019;91:195-225.

48. Rossi-Izquierdo M, Gayoso-Diz P, SantosPérez S, Del-Río-Valeiras M, Faraldo-García A, Vaamonde-Sánchez-Andrade I,et al. Vestibular rehabilitation in elderly patients with postural instability: reducing the number of falls-a randomized clinical trial. Aging Clin Exp Res. 2018;30:1353-61. 